\title{
Vector-Borne Viral Fevers and Eye: An Observational Study on Ocular Manifestations
}

\author{
Authors \\ Prateek Koul ${ }^{1}$, Vijayta Gupta ${ }^{2}$ \\ ${ }^{1}$ Senior Resident, VCGS Government Medical College, Srinagar, Uttarakhand \\ ${ }^{2}$ Senior Resident, Department of Ophthalmology, Government Medical College Jammu \\ *Corresponding Author \\ Vijayta Gupta
}

\begin{abstract}
Aims: To study the pattern of ocular manifestations in vector borne viral fever cases admitted in a tertiary care hospital.

Study Design: Descriptive observational case series study.

Place and Duration of Study: Department of Ophthalmology and Medicine VCGS Government Medical College, Srinagar, Uttarakhand between March 2018 to October 2018.

Methodology: Fifty patients admitted in medical wards having clinical features of chikungunya and dengue with serological confirmation during the peak season of viral infections between March 2018 and October 2018 were included in this study. They were subjected to ophthalmological evaluation and investigations.

Results: The eye complications recorded in these patients were periorbital ecchymosis, conjunctivitis, subconjuctival, hemorrhage, episcleritis, scleritis, anterior uveitis, posterior uveitis, retinal vasculitis, macular edema. These patients were having significant thrombocytopenia and leucopenia. Visual prognosis was good with treatment.

Conclusion: Lot of patients in the study group had various ocular findings associated with these viral fevers.
\end{abstract}

Keywords: [Dengue, Chikungunya, Conjuctivitis, thrombocytopenia].

\section{Introduction}

The two major vector-borne viral fevers detected in the north India are dengue and chikungunya and transmitted through the bite of infected female Aedes Aegypti or Aedes albopictus mosquito, which are domestic day biting ones with preference to human blood.

Dengue is caused by one of the genus Flavivirus of the family Flaviviridae. The disease spectrum ranges from mild influenza like illness to life threatening hemorrhagic fever/shock syndrome with epistaxis, bleeding gums and bleeding tendencies anywhere in the body. Chikungunya is caused by Alphavirus of the family Togaviridae.

Symptoms are fever of abrupt onset, headache, myalgia, arthralgia, nausea, vomiting and maculopapular rashes. The various ocular manifestations have been reported from different countries which may vary with each outbreak.

The aim of the study was to fund out the pattern of ocular manifestations in vector borne viral fever cases reported in a tertiary care hospital in north India. 


\section{Material and Methods}

This was a descriptive observational case study. Patients admitted to the medical wards of a tertiary care center with a clinical diagnosis of chikungunya fever, dengue fever and dengue hemorrhagic fever during the peak season between March and October 2018 were included in this study. Patients with a serological diagnosis of chikungunya and dengue fever studied. Patients were analyzed for the demographic features, ocular and systemic symptoms, ocular signs and laboratory investigations. Laboratory investigations included complete hemogram and Elisa tests for immunoglobulin M (IgM) dengue and chikungunya.

Patients with other infectious fevers including leptospirosis, tuberculosis, human immunodeficiency virus and patients with pre existing ocular diseases both congenital and acquired were excluded from the study. Symptomatic patients were followed up in ophthalmology OPD. All other patients were asked to attend Ophthalmology department if the develop ocular symptoms over a period of 1 month.

All these patients were subjected to complete ophthalmological evaluation including slit lamp examination, tonometry and dilated fundus examination with indirect ophthalmoscope and 90D lens. Functional examinations such as bestcorrected visual acuity with snellens chart, color vision, visual field charting was done.

Those patients with vision-threatning complications such as optic neuritis, retinal vasculitis and macular edema were treated with systemic prednisolone $1 \mathrm{mg} / \mathrm{kg}$ bodyweight in tapering doses. Conditions such as anterior uveitis, episcleritis, scleritis and conjunctivitis were treated as per guidelines.

The study was conducted after obtaining informed written consent from patients. There were no drug trials and other treatment modalities inflicted upon the patients.

\section{Results}

50 patients admitted in the medical wards having clinical features of chikungunya or dengue were analyzed for the presence of ocular manifestations. Patients with positive IgM chikungunya/dengue were studied. Statistical analysis was done with software SPSS-16 version. The age of the patients ranged from 10 to 70 years, mean age being 36 with standard deviation of 16.Males were affected more than females $(52 \%$ $\mathrm{n}=26$ ). both eyes were affected in 14 patients. IgM dengue and Ig M chikungunya were found to be positive in 25 and 15 patients respectively.10 patients were positive both for dengue and chikungunya indicating mixed infection. Out of 50 confirmed patients evaluated $30 \quad(60 \%)$ had associated ocular signs.

Time of onset of ocular symptoms varied from 1 day to 1 week in majority of cases, 18 out of 30.Ocular symptoms started along with fever in 6 patients. In 4 patients with isolated chikungunya positivity, ocular symptoms started 4 weeks after the onset of fever. The predominant systemic symptoms were myalgia, rashes, joint pain and headache. The ocular signs varied from lid involvement to anterior and posterior segment involvement Tab 1.Periorbital ecchymosis was found in two patients. Anterior and posterior segment signs were present in 21 and 3 patients, respectively.

Thrombocytopenia was noticed in $80 \%(n=40)$. Leukopenia was detected in 10 patients out of 50.Other investigations are given in Tab 2.

Table 1: Ocular Signs

\begin{tabular}{|l|c|}
\hline OCULAR SIGNS & CONFIRMED CASES (\%) \\
\hline Periorbital ecchymosis & $2(4)$ \\
\hline Subconjuctival Hemorrhage & $4(8)$ \\
\hline Conjuctivitis & $8(16)$ \\
\hline Keratitis & $1(2)$ \\
\hline Episcleritis & $2(4)$ \\
\hline Scleritis & $2(4)$ \\
\hline Iridocyclitis & $6(12)$ \\
\hline Trabeculitis & $1(2)$ \\
\hline Posterior uveitis & $1(2)$ \\
\hline Retinal vasculitis & $1(2)$ \\
\hline Macular edema & $2(4)$ \\
\hline
\end{tabular}


Table 2: Investigation Results

\begin{tabular}{|l|c|}
\hline INVESTIGATIONS & CONFIRMED CASES(\%) \\
\hline Leucopenia & $10(20)$ \\
\hline IgM dengue & $25(50)$ \\
\hline IgM chikungunya & $15(30)$ \\
\hline Both IgM positive & $10(20)$ \\
\hline Right Eye involved & $9(18)$ \\
\hline Left eye involved & $7(14)$ \\
\hline Both eyes involved & $14(28)$ \\
\hline Thrombocytopenia & $40(80)$ \\
\hline
\end{tabular}

Table 3: Ocular Features in Isolated Dengue

\begin{tabular}{|l|c|}
\hline $\begin{array}{l}\text { OCULAR FEATURES IN } \\
\text { DENGUE CASES (N=25) }\end{array}$ & N(\%) \\
\hline Periorbital ecchymosis & $2(8)$ \\
\hline Subconjuctival hemorrhage & $2(8)$ \\
\hline Acute conjuctivitis & $8(32)$ \\
\hline Scleritis & $2(8)$ \\
\hline Keratitis & $1(4)$ \\
\hline Acute Iridocyclitis & $2(8)$ \\
\hline
\end{tabular}

\section{Discussion}

The onset of ocular problems coincides with the resolution of fever and appearance of thrombocytopenia, usually ranges from 1 to 2 weeks $^{[1][2]}$. Late onset was seen in 4 patients with isolated chikungunya positivity ${ }^{[3]}$.

In this study, myalgia $(90 \%, \mathrm{n}=45)$ was the predominant general symptom followed by joint pain $(80 \%, \mathrm{n}=40)$ and headache $(78 \%, \mathrm{n}=39)$.

Significant ocular symptoms were redness in 16 followed by retro orbital pain in 12,blurred vision in eight, photophobia in six, and discharge in two patients. Characteristic retro orbital pain associated with dengue and chikungunya has been reported previously in various studies ${ }^{[1]}$. Both eyes were affected in 12 patients. When these patients were evaluated, 30 had associated ocular signs /symptoms rest 20 were asymptomatic, which is matching with previous studies ${ }^{[4]} .25$ patients had isolated IgM dengue and 15 had positive IgM for chickungunya.10 were positive for both dengue and chikungunya. In isolated dengue patients 12 out of 25 developed ocular problems within a week in form of periorbital ecchymosis ${ }^{[3]}$, acute conjunctivitis, subconjuctival hemorrhage, scleritis, keratitis and iridocyclitis Tab 3.

Probable pathogenic factors responsible for this could be viremia and thrombocytopenia. In some epidemics, bleeding complications are more common. In this study, acute conjunctivitis was the most common presentation in dengue patients when compared to previous studies these were subconjuctival hemorrhage, secondary marked thrombocytopenia ${ }^{[4]}$.

Anterior uveitis needs special mention here. There was marked pigment release in the anterior chamber with pigmented KPs while posterior synechiae were insignificant. This was similar to previous reports of chikungunya iridocyclitis ${ }^{[1]}$. Late onset of ocular symptoms in chikungunya, postulates an immune -mediated mechanism rather than acute viremia. Compared to previous study ${ }^{[3]}$, we did not have any patient of retinitis in pure chikungunya infection. In their study out of the 9 patients, 1 had episcleritis, 5 iridocyclitis and 3 had retinitis. Furthermore in their study ocular problems started 4 weeks after onset of fever. Although there were no specific changes of iridocyclitis that were diagnostic of fever, the retinal changes were consistent with retinitis. All patients recovered from the infection with relatively good vision ${ }^{[3]}$. Out of 10 patients with mixed infection 4 showed ocular problems.1 patient each had acute conjunctivitis, episcleritis. scleritis and retinal vsulitis with macular edema. Moreover all of them developed symptoms in the first week itself.1 patient with nodular scleritis needed immunosuppressive therapy. Majority of the patient had normal visual acuity and those presented with blurring of vision due to posterior segment pathologies improved after treatment. The cause for defective vision was iridocyclitis in 6 patients. Posterior uveitis and retinal vasculitis were responsible for the same in 1 each. One patient had defective vision due to macular edema. The postulated theory behind these inflammatory responses is that vasoactive and inflammatory mediators cause capillary leakage and produce macular edema and breakdown of blood aqueous barrier resulting in periphlebitis. ${ }^{[4]}$ Blurring of vision typically coincides with the incidence of thrombocytopenia and occurs a week after onset of fever ${ }^{[5]}$. 
Chikungunya virus infection has recently been reported to cause different ocular manifestations such as anterior uveitis, episcleritis, panuveitis, optic neuritis, sixth nerve palsy, retrobulbar neuritis, retinitis, vitiritis, CRAO and exudative retinal detachment ${ }^{[6]}$.

Regarding hematological evaluation, thrombocytopenia was noticed in $80 \%$ and leucopenia in 10 patients. Presence of thrombocytopenia was statistically significant with $\mathrm{p}=0.001$. All 10 patients with mixed infections had thrombocytopenia. In spite of that hemorrhagic complications were less in this study when compared to another study ${ }^{[7]}$ where most common ophthalmic signs were macular edema in $76.9 \%$ and macular hemorrhage in $69.2 \%$.

Since this study was hospital based with small sample size in addition PCR study was not done due to cost issues in our study population. These factors can be considered as limitations.

\section{Conclusion}

The main ocular manifestation associated with vector-borne viral fever in gharwal region of uttarakhand India, included periorbital ecchymosis, conjunctivitis, subconjuctival hemorrhage, episcleritis, scleritis, anterior uveitis, retinal vasculitis and macular edema. The visual prognosis was good after treatment with systemic steroids in indicated cases. Statistically significant thrombocytopenia was present in patients having ocular manifestations. Hence, thrombocytopenia can be an indicator for the treating physician for an ophthalmological evaluation of the patient.

\section{Conflict of interest: None \\ Funding: None}

\section{References}

1. Khairallah M, Jelliti B, Jenzeri S.Emergent infectious uveitis. Middle East Afr J Ophthalmol 2009;16:225-38..

2. Shikha R, Ratnesh R. Ocular Manifestations of emerging viral diseases in India. Delhi J Ophthalmol 2014;24:2756.

3. Mahendradas P,Ranganna SK,Shetty R, Balu R, Narayana KM,Babu RB et al. Ocular manifestations associated with chikungunya. Ophthalmology 2008;115: 287-91.

4. Kapoor HK, Bhai S,John M,Xavier J. Ocular manifestations of dengue fever in an east Indian epidemic. Can J Ophthalmol 2006;41:741-6.

5. Mohindra VK, Kumari A. Unilateral optic neuritis associated with dengue fever.DJO 2013;23:289-91.

6. Lalitha $\mathrm{P}$, Rathinam S,Banushree K, Maheshkumar S, Vijayakumar R, Sathe P. Ocular involvement associated with an epidemic outbreak of chikungunya virus infection. Am J Ophthalmol 2007;144:552-6.

7. Chan DP, Teoh SC,Tan CS,Nah GK, Rajagopalan R, Prabhakaragupta MK, et al. Ophthalmic complications of dengue. Emeg Infect Dis 2006;12:285-9. 